\title{
Research summary of the Micro Grid Energy Management System Control Structure
}

\author{
Xiaocen $\mathrm{Cai}^{1,}$, , Dongtao Wang ${ }^{2, \mathrm{~b}}$ and Zhilong $\mathrm{Gao}^{3, \mathrm{c}}$ \\ ${ }^{1}$ Tianjin University of Technology and Education: Joint Lab of Information Sensing \& Intelligent \\ Control,China \\ ${ }^{2}$ Tianjin University of Technology and Education: Joint Lab of Information Sensing \& Intelligent \\ Control,China \\ ${ }^{3}$ Tianjin University of Technology and Education: Joint Lab of Information Sensing \& Intelligent \\ Control,China \\ acxcandtoni@163.com, bdongtwang@sohu.com, c'gz190507@163.com
}

Keywords: Micro power grid; Energy management; Control structure

\begin{abstract}
With the continuous development of micro grid technology, the power energy management system has gradually become a research hotspot. Micro grid energy management system can be divided into two control structures, centralized control and decentralized control.The multi-agent system is the best choice for these two kinds of structure. And then we introduced the multi-agent system building. Finally this paper summed up the problems that need to be solve in micro grid energy management: renewable energy and controllable load more uncertainty problem, the optimization of energy storage technology cooperation and joint scheduling problem, the micro grid communication design of energy management system and network security problem.
\end{abstract}

\section{Introduction}

With the establishment and development of micro power grid project to match the energy management system has gradually become a research hotspot. Micro grid energy management system is an energy management software which includes power optimization scheduling, load management, real-time monitoring and automatic micro grid synchronization and other functions. This paper analyzes the micro grid energy management system control structure, multi-agent system are introduced in the application of the micro energy management system. It gives an example of building multi-agent energy management system. In the end, this paper points out some technical problems existing in the current study, further research directions are presented.

\section{The micro grid power system control structure}

From the point of the control structure of micro grid energy management system, micro grid can be divided into centralized control and decentralized control.

1.1 The centralized control structure

The centralized control, the power grid to the control of all power generation units as well as the energy storage is united by a central control unit to complete. Each unit in the micro power grid will be delivered to the master in their respective operation information unit, on the premise of guarantee the stability of system, both the economic operation, main control unit to control signals through high speed communication network to the micro power grid within each unit. This control scheme is simple and efficient; and the adjusting effect quickly and facilitate global network.But because all of the information system to focus on, so you need to have an efficient controller to deal with huge data and the center of global information transmission in the network, which mainly depends on the center controller. Once it appears single point of failure, control will fail.In addition, the control and operation information is totally dependent on high-speed communication networks, communication distance and cost constraints, communication system failure will cause the whole micro power grid paralysis, reduces the system reliability. In addition, once the control framework is determined, the 
structure of the network are unable to change. When there is a new power source or load to detail power grid, it need to change control structure. So this method is only suitable for space truss structure is fixed, simple structure, small micro grid system, the structure is complex, load growth in demand for more micro grid information will appear in the disaster and powerless.

\subsection{Distributed control structure}

Decentralized control is another kind of micro grid energy management system control mode. Decentralized control manner \& each element in the micro grid is controlled by local controller, each local monitoring operation condition of the source of micro controller, and through the communication network to communicate with other local controller. Local controller does not need to receive the central controller control instruction, have decide the micro source controlled by the power of the running status. Due to the local controller only need communication with neighboring devices, the amount of information transmission less than centralized control) the amount of calculation is share to each local controller, reduces the workload of the central controller. Central controller in the distributed control structure is mainly responsible for transfer of the upper system load and price information, as well as in the case of emergency or failure from the system level, the manipulation of the local controller.

Centralized and decentralized control manner are the central controller and the local controller, just distributed control weakening the dominant function of the central controller, through enhancing peripheral communication, scatter control power to the local controller, central controller and the local controller even distribution center plays a certain role in the management system. Multi-agent system can better simulate the role of a particular function.

1.3 Multi-agent in the application of traffic management system

Multi-agent System (MAS, Multi - Agent System) is a kind of distributed autonomous System, composed of multiple Agent, the Agent together, through the mutual coordination and cooperation between each Agent, solve common complex problems.General Agent structure includes four modules: information processor, object module, sensor module, communication mechanism .Its basic structure is shown in Fig.1.

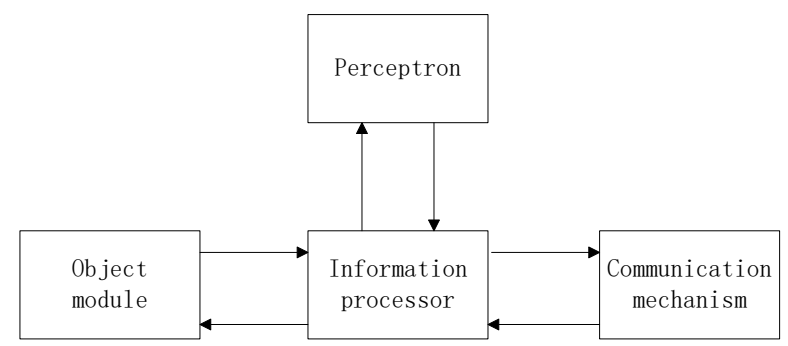

Fig.1. The basic structure of the Agent

The Agent through the four parts to function.Through the sensor module for the environment information, update the internal data;Communication mechanism specifies the communication mode between the Agent and interactive information, complete with other Agent cooperation;Object module storage Agent need to complete user tasks and operation steps, etc.;Information processor as the core part of the information was obtained from the other three modules and processing, make intelligent feedback and send back the information to the other three modules related components.

Application in micro grid multi agent system is the single agent together.Commonly used multiple proxy connection structure are: distributed structure, hierarchical structure and mixed structure, they are as shown in Fig2- Fig.4.

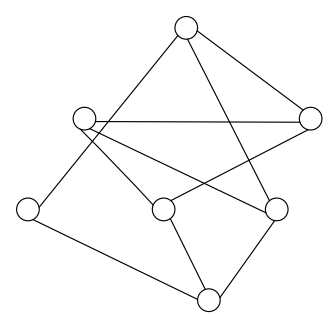

Fig.2 distributed structure 


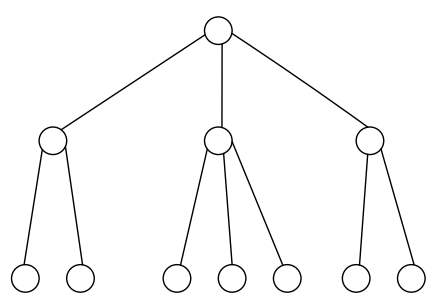

Fig.3 layered structure

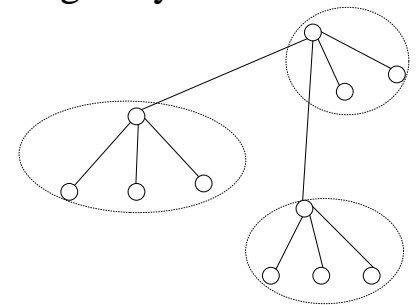

Fig.4 mixed structure

\section{The establishment of multi-agent energy management system}

The multi-agent energy management system structure

Control system based on MAS, in accordance with the"dominant Agent, node Agent, Agent controlled unit" of the three layer architecture design, as shown in Fig.5.

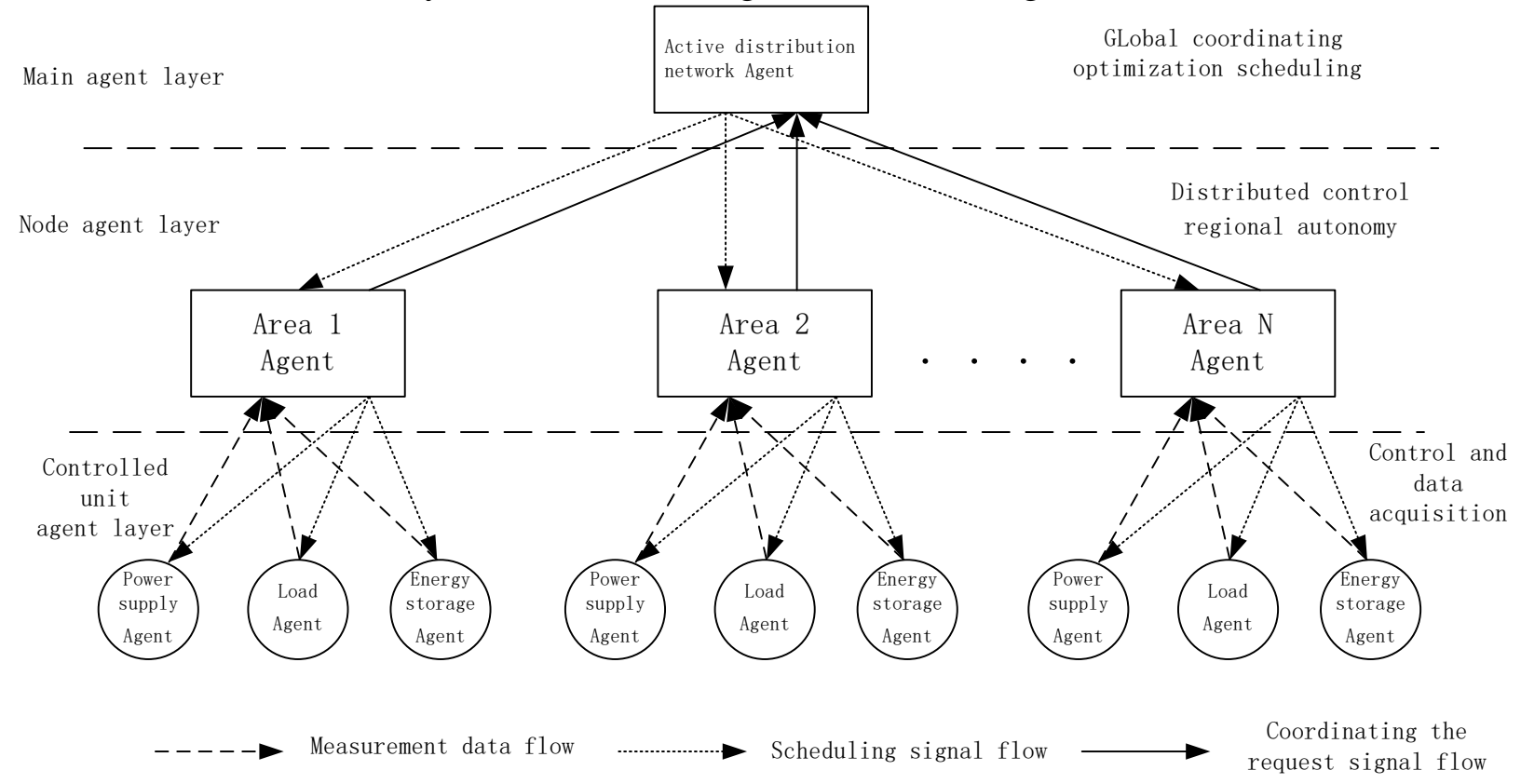

Fig.5 the multi-agent control structure

1) the main Agent, on the one hand, according to the entire network optimization goal setting area excitation signal, on the other hand is responsible for the entire network security constraints checking.

2) node Agent, response dominant Agent of excitation signal, based on their operation target (cost minimum target), achieve the equilibrium of regional autonomy in internal energy.

3) Agent controlled unit, used for network resources such as distributed generation, such as flexible load, energy storage object behavior of the Agent, the response node Agent control instruction.

Figure 5 layer nodes can be extended according to the voltage grade, expanded into a layer 2 or more layers, different levels of optimization implement active controlling of hierarchical partitioning of the distribution network.

The above architecture can support different control modes: 
1) regional autonomy: the regional internal energy balance, this pattern is similar to micro network mode, but the main difference is that the node Agent in the region of the main Agent of excitation signal coordinated guidance, to realize the energy balance of the optimization goal of self-government.

2) global cooperation: according to the main Agent of global optimization scheduling, power interaction between multiple regions to achieve cross-regional coordination of the entire network energy.

2.2 Energy management system of multi-agent modeling

2.2.1 Load cell Agent

Starting from the load can be regulatory, load can be divided into rigid load and flexible load:

$$
P L(t)=P L f(t)+P L d r(t)
$$

In the type: rigid load PLf (t) is characterized by the load change which is not affected by the electricity price fluctuation, it can be expressed as don't change with the outside world at time constant;Flexible load PLdr (t) of electricity behavior has great flexibility, which is influenced by the electricity price and other parameters affecting the behavior of electricity load factor (such as temperature, sunshine intensity, etc.).

2.2.2 Agent storage unit

Energy storage devices are being limited by the maximum storage capacity, and in order to prolong the service life of the battery, don't allow the charge.

Energy storage model is

$$
E t=E t(t-1)+\Delta T P \operatorname{ch}(\mathrm{t}) \eta \operatorname{ch}-\Delta T P \operatorname{dis}(\mathrm{t}) \eta \operatorname{dis}
$$

The constraints are

$$
\left\{\begin{array}{l}
\text { Ec } \operatorname{SOCmin} \leq \mathrm{E}(\mathrm{t}) \leq \mathrm{Ec} \text { SOCmax } \\
0 \leq \mathrm{P} \operatorname{ch}(\mathrm{t}) \leq \mathrm{Pc}, \max \\
0 \leq \mathrm{Pdis}(\mathrm{t}) \leq \mathrm{Pd}, \text { max }
\end{array}\right.
$$

In the type: $\mathrm{E}(\mathrm{t})$ is the total power of the $\mathrm{t}$ slots; $\eta \mathrm{ch}$ and $\eta$ dis is charging and discharging efficiency; Ec is rated capacity; SOCmin and SOCmax are respectively minimum/maximum charging status, value range is $(0,1]$; $\mathrm{Pd}$, Max and $\mathrm{Pc}$, Max are respectively put/charging maximum power.

2.2.3 Agent power generation unit

Power generation unit is divided into controllable power and uncontrolled generation of electricity.In the type controllable power including power plants, gas turbine and fuel cells, etc. Uncontrolled in the type power mainly renewable energy power generation, with wind power (wind turbine, WT) and photovoltaic cells (photovoltaiccell, PV) power generation is given priority to, with maximum power point tracking (maximumpower point tracking, MPPT) control mode, ensure maximum use of renewable energy, is not scheduling unit, not involved in autonomous coordination control.

The constraints of distributed generation are

Pdg, $\min \leq \operatorname{Pdg}(\mathrm{t}) \leq \mathrm{Pdg}, \max$

In the type: Pdg, Max and Pdg, min are respectively the upper and lower limits of the efforts of distributed generation.

2.2.4 Node Agent

Node Agent optimization goal is to ensure maximum use of renewable energy power generation under the premise of node Agent response leading Agent excitation signal, controllable unit in the 
node Agent autonomy for scheduling, realize the user's operation cost minimum.It is power balance constraints, contact circuit, voltage constraints.

\subsubsection{Leading Agent}

Main Agent distributed function mainly includes the excitation signal and system security constraint checking.The former for the node Agent autonomy behavior intervention, which realize the autonomy of each node Agent check results.Main Agent used for excitation signal is a set of constraints node curve, the actual meaning of the curve reflects the dominant Agent running on the node expectations, it can be set by different meanings of target curve for differentiation of different nodes independently optimize control: such as global goal is to realize the peak load cutting and can set the excitation signal to grid electricity price signal.Set up according to the load forecasting load peak power grid electricity price is higher, low power grid electricity price low to guide the node Agent active response.As the global safety goal is to use the security constraint optimization signal (reactive power/voltage limit, etc.). According to the data optimization results, set the limit, the active node Agent for a response.In the complicated distributed network to achieve maximum minimum traffic and information transmission.

\subsection{Solution algorithm of multi-agent energy system}

Multi-agent energy management system to solve the multi-objective optimization problem, often can be thought of as often by weighted objective function can be converted toSingle objective optimization problem, using the single objective optimization method.Also can use multi-objective optimization method, such as the dominant multi-objective genetic algorithm.

At present most of the micro grid model does not consider the energy management system trend of constraint, using the method of solving mixed integer linear programming method, dynamic programming method, genetic algorithm and particle swarm optimization algorithm, ant colony algorithm, intelligent algorithm and expert system based on rule algorithm. The mixed integer linear programming method by using the optimization model of nonlinear function is converted into linear function, thus the optimization problem into a mixed integer programming problem. USES the mature professional software solution, usually has high calculating precision and faster speed, to consider the trend of constraint model, optimal power flow is generally to join in the process of calculation or power generation method and power flow calculation subroutine.Due to the nonlinear constraints to soar, usually using genetic algorithm and particle swarm optimization intelligent algorithm is optimized.

\section{The micro grid energy problem and development direction of the management system}

At present, the micro grid energy management mainly adopts centralized control, with mature technology, distributed control will gradually become the development direction of micro grid power management control structure.Decentralized control enables the distributed power to plug and play, any distributed power or energy storage equipment at any time can be connected to the power grid, dramatically increase the flexibility of the electricity.

Micro grid energy management system for the use of micro grid convenience and efficiency play an important role.Due to the particularity of micro power grid, the grid energy management still faces a series of challenges, mainly has the following three aspects.

1. In the micro grid renewable energy sources such as wind power, photovoltaic output is affected by the natural environment, have the characteristics of intermittent, and poor volatility and predictability.The design of micro grid energy management system need to consider the impact of these random factors.

2. A variety of energy storage technology optimization and energy storage system of joint scheduling will become a micro grid energy management difficulty.

3. Micro grid energy management system of communications network construction and communication security is a problem worth studying.

\section{Summary}


Micro grid energy management is an important research content in the grid technology, with the continuous development of micro power grid and the expansion of scale, micro grid energy management will be in the control structure, communication design and a series of problems need to be solved. This paper summarizes the control structure of energy management system, and the energy management system of multi-agent technology research hot spots are introduced.For now the research work have been summarized existing problems and difficulties, the direction of further research are pointed out.

\section{References}

[1] Pu tianjiao, Liu Kewen and Li Ye: Proceedings of the csee Vol.35 (2015), p. 1865

[2] Ming Ding, Wei Yang and Yingyuan Zhang: Electric Power Automation Equipment Vol.29 (2009), p. 16-20.

[3] W Su, J Wang: The Electricity Journal Vol.25 (2012), p. 45-60

[4] Darabi Z, Ferdowsi M: IEEE Transactions on Sustainable Energy ,Vol.2 (2011), p. 501-508

[5] Ming Ding, Kui Luo and Rui Bi: Journal of coordinated control strategy of power system automation Vol.37 (2012), p. 1-8

[6] Lasseter R, Eto J and Schenkman B: IEEE Transactions on Power Delivery Vol.26 (2011), p. 325-332 